\title{
Multimodal therapeutic assessment of peripheral nerve stimulation in neuropathic pain: Five case reports with a 20-year follow-up
}

\author{
Ron Kupers ${ }^{\mathrm{a}, \mathrm{b}, *}$, Koen Van Laere ${ }^{\mathrm{c}}$, Frank Van Calenbergh ${ }^{\mathrm{d}}$, Jan Gybels ${ }^{\mathrm{d}}$, Patrick Dupont ${ }^{\mathrm{c}, \mathrm{f}}$, Annelies Baeck ${ }^{\mathrm{b}}$, \\ Leon Plaghki ${ }^{\text {e }}$
}

a Institute for Neuroscience and Pharmacology, Panum Institute, University of Copenhagen, Copenhagen, Denmark

${ }^{\mathrm{b}}$ PET Unit, Rigshospitalet, University of Copenhagen, Copenhagen, Denmark

${ }^{\mathrm{c}}$ Department of Nuclear Medicine, University Hospital Gasthuisberg, Katholieke Universiteit Leuven, Leuven, Belgium

${ }^{\mathrm{d}}$ Department of Neurosurgery, University Hospital Gasthuisberg, Katholieke Universiteit Leuven, Leuven, Belgium

e Unité de Réadaptation et Médecine Physique, Faculté de Médecine, Université Catholique de Louvain, Brussels, Belgium

${ }^{\mathrm{f}}$ Medical Imaging Research Center and Laboratory for Cognitive Neurology, Katholieke Universiteit Leuven, Leuven, Belgium

\section{A R T I C L E I N F O}

\section{Article history:}

Received 18 March 2010

Received in revised form 23 May 2010

Accepted 16 June 2010

Available online $\mathrm{xxxx}$

\section{Keywords:}

Neuropathic pain

Neuromodulation

Quantitative sensory testing

Laser-evoked potentials

Brain imaging

\begin{abstract}
A B S T R A C T
Neuropathic pain following peripheral nerve lesion is highly resistant to conventional pain treatments but may respond well to direct electrical peripheral nerve stimulation (PNS). In the 1980s, we treated a series of 11 peripheral neuropathic pain patients with PNS. A first outcome assessment, conducted after a 52-month follow-up, revealed that the majority of the patients were significantly improved. Here, we present the results of a second and more comprehensive follow-up, conducted after more than 20 years of PNS usage. Of the six patients still using PNS, five participated in a multimodality assessment of the long-term efficacy of PNS. Home evaluations showed reduced pain ratings and improved quality-of-life during active periods of stimulation. Quantitative sensory testing confirmed the neuropathic character of the pain complaints. PNS had no significant overall effect on tactile detection, cool, warmth, cold pain and heat pain thresholds. Laser-evoked potentials showed an enlarged N2-P2 complex during active PNS. Positron Emission Tomography revealed that PNS decreased activation in the pain matrix at rest and during thermal stimulation. PNS led to increased blood flow not only in primary somatosensory cortex, but also in anterior cingulate and insular cortices, suggesting that besides activation of the dorsal column lemniscal system, other mechanisms may play a role in its analgesic effects. These data show that PNS can provide truly long-term pain relief in carefully selected patients and they provide some objective quantitative data in support of this. They encourage the planning of future prospective studies in a larger cohort of patients.
\end{abstract}

(C) 2010 European Federation of International Association for the Study of Pain Chapters. Published by Elsevier Ltd. All rights reserved.

Cite this article as: Ron Kupers, Koen Van Laere, Frank Van Calenbergh, Jan Gybels, Patrick Dupont, Annelies Baeck, Leon Plaghki. Multimodal therapeutic assessment of peripheral nerve stimulation in neuropathic pain: Five case reports with a 20-year follow-up. Eur J Pain xxx (2010) xxx-xxx [doi:10.1016/ j.ejpain.2010.06.015]

Full text available online on ScienceDirect ${ }^{\circledR}$, www.sciencedirect.com.

\section{Introduction}

Chronic neuropathic pain following injury to a peripheral nerve is highly resistant to both conventional pain therapy and neurosurgical treatment at the injury site (Gybels and Sweet, 1989). Based upon the theoretical predictions of the gate control theory

\footnotetext{
* Corresponding author at: Institute for Neuroscience and Pharmacology, Panum Institute, University of Copenhagen, Blegdamsvej 3B, DK-2200 Copenhagen, Denmark. Tel.: +45 3545 6890; fax: +45 35458949 .

E-mail address: ron@pet.rh.dk (R. Kupers).
}

(Melzack and Wall, 1965), Wall and Sweet (1967) showed that electrical stimulation of the large myelinated fibers of a peripheral nerve can alleviate pain. This led to the first clinical trials with fully implanted devices in selected patients with neuropathic pain due to peripheral nerve lesions (Sweet, 1976). Direct electrical peripheral nerve stimulation (PNS) through fully implantable electronic devices was demonstrated to be relatively successful in the short and intermediate term (Mobbs et al., 2007). However, contrary to spinal cord stimulation (SCS) or deep brain stimulation (DBS), PNS never became very popular. Explanations for this lack of enthusiasm include technical and surgical difficulties to use 
wire-type or paddle-type electrodes (originally designed for SCS) in close contact with peripheral nerves, lack of interest by industry to develop adequate electrodes for this relatively small patient population and lack of reimbursement by health insurance companies. Slightly unexpectedly, interest in PNS surged in recent years within the neurosurgical community, in particular for the treatment of persistent craniofacial pains (Burns et al., 2009; Slavin and Burchiel, 2000) but also for other forms of neuropathic pain (Slavin (2008), for a review).

In 1988, we performed a first systematic evaluation of the clinical outcome in a series of 11 patients with peripheral neuropathic pain treated with PNS. The majority of patients were significantly improved after an average follow-up of 52 months (Gybels et al., 1990; Gybels and Van Calenbergh, 1990). A quite remarkable finding was that several patients continued to benefit pain relief while progressively reducing frequency of stimulation. The aim of the present study was to evaluate the clinical efficacy of PNS at a much longer follow-up period and to relate it to "objective" measures of peripheral and central pain processing. We combined home assessments of pain and quality-of-life, quantitative sensory testing (QST), laser-evoked potentials (LEPs) and Positron Emission Tomography (PET). QST was performed to objectify the neuropathic character of the sensory complaints. LEPs were used to read the functional integrity of the afferent nociceptive system (Mouraux and Iannetti, 2009), whereas PET was chosen to investigate whether the subjective reports of pain relief are supported by altered activation patterns within the pain matrix. We hypothesized that: (1) the QST data will confirm the presence of a neuropathic pain condition; (2) responses, such as LEPs, to painful (but not to innocuous) stimuli will be modified by PNS; and (3) PET data will show reduced activity within the pain matrix during active PNS together with increased activity within the somatosensory cortex and pain modulatory areas such as the anterior cingulate cortex.

The clinical data on the effects of PNS on pain ratings and quality-of-life have been described in a previous report (Van Calenbergh et al., 2009).

\section{Methods}

\subsection{Participants}

All patients implanted with a PNS device at the University Hospital of Leuven were considered as candidates for participation in the current study. Of the original 11 patients from the first follow-up study, one had died in the meantime and four had stopped using PNS because of hardware failures. These patients were treated with SCS instead. Of the six patients still using PNS, one refused to participate in the study because of the geographical distance between his home and the hospitals where the examinations were going to be carried out. We therefore finally studied five patients (three females) who still actively used PNS to treat their pain. Patients' characteristics are summarized in Table 1 . We provide a detailed case description in Online supplementary section. All patients provided written informed consent and the protocol was approved by the local ethics committee of the University of Leuven.
Patients were first invited for an interview by one of the neurosurgeons (FVC, JG). The purpose of this interview was to gather information about the use of PNS, its therapeutic effect and the duration of the analgesic after-effect after a treatment session. This information was used to plan the QST, LEP and PET sessions. Patients were explained the purpose of the study and they were made familiar with the questionnaires and pain rating forms they had to fill in at home. Once a patient accepted to participate, he/she was randomized to start with either the "PNS-ON" or "PNS-OFF" condition.

The normative data for QST and LEPs come from an age- and sex-matched group of healthy volunteers recruited among colleagues and relatives, who have no history or symptoms of neurological disorders or abnormalities.

\subsection{Home-ratings of pain and daily activities}

Patients were asked to fill in a questionnaire relating to their pain and daily activities three times daily (morning, afternoon and evening) during 3 days in both the PNS-ON and PNS-OFF conditions. The questionnaire used is similar to the one officially used and approved by the Belgian health care authorities for assessing the efficacy of SCS during the pre-implantation phase (Kupers et al., 1994). Pain was measured using VAS and verbal rating scales (Van Calenbergh et al., 2009). At the end of the 3-day evaluation period, participants filled in a questionnaire relating to their average pain and daily activities experienced over the full 3-day assessment period (Van Calenbergh et al., 2009).

\subsection{Quantitative sensory testing}

The QST examination comprised an assessment of both the thermal and tactile modalities. All patients were tested twice with an interval of at least one week between the two sessions. QST was assessed during a period of normal usage of PNS and after patients had refrained from using PNS and their sensory symptoms (pain and allodynia) had returned. When patients were tested in the "PNS-ON" period, they were asked to refrain from stimulation at least $1 \mathrm{~h}$ before the start of the measurements. In order to preserve blinding, patients were instructed not to inform the investigator (LP) performing the QST and LEP measurements about the state of PNS during testing.

\subsubsection{Thermal testing}

For thermal testing, a Peltier-based temperature feedback-controlled system was used (Medoc, TSA-II; Haifa, Israel) with a probe size measuring $30 \times 30 \mathrm{~mm}$. We measured cool and warmth detection thresholds and cold pain and heat pain thresholds using the method of limits. Thermal testing was performed in the painful area and in the contralateral mirror area. The order of testing across the two regions was randomized. Measurement of cool detection threshold (CDT) and warmth detection threshold (WDT) always preceded the pain threshold measurements. For cool and warmth detection, the thermode was placed on the skin at baseline skin temperature. The temperature slightly increased or decreased $(0.5 \mathrm{deg} / \mathrm{s})$ and the subject pressed a button when

Table 1

Summary patient characteristics.

\begin{tabular}{|c|c|c|c|c|c|c|c|c|c|}
\hline \multirow[t]{2}{*}{ Patient } & \multirow[t]{2}{*}{ Age } & \multirow[t]{2}{*}{ Sex } & \multicolumn{5}{|l|}{ Pain complaints } & \multicolumn{2}{|c|}{ Peripheral nerve stimulation } \\
\hline & & & Cause & Age onset & Years & Region & Tactile allodynia & Stimulated nerve & Years of usage \\
\hline VBM & 64 & $\mathrm{~F}$ & Amputation & 14 & 50 & Left ring finger & Severe & Ulnar left & 18 \\
\hline DRR & 66 & $\mathrm{~F}$ & Amputation & 7 & 59 & Right ring finger & Severe & Ulnar right & 29 \\
\hline DBH & 60 & M & Burn injury & 27 & 33 & Left hypothenar & No & Ulnar left & 26 \\
\hline HUV & 60 & M & Cat bite & 40 & 20 & Right thumb & Medium & Median right & 18 \\
\hline VDB & 54 & $\mathrm{~F}$ & Traumatic fall & 26 & 28 & Left thenar & Mild & Median left & 25 \\
\hline
\end{tabular}


he/she felt the sensation of cool or warmth. The procedure was repeated four times for the two body regions tested. Thereafter, pain threshold measurements were performed, always starting with the heat pain threshold (HPT). Temperature started at baseline and slowly increased $(0.5 \mathrm{deg} / \mathrm{s})$. Patient pressed a button at the moment he/she felt a painful sensation. The procedure was repeated three times for the two body regions. Thereafter, cold pain threshold (CPT) was assessed in the same manner.

\subsubsection{Tactile testing}

For tactile testing, a set of Semmes-Weinstein filaments was used. Tactile thresholds were assessed in the painful area and in the contralateral mirror area. We used a double interlaced staircase algorithm adapted from Dyck and co-workers (1993). This has the advantage that the patient cannot predict (as in a classical staircase method) whether the upcoming stimulus will be more or less intense than the preceding one. This method is therefore very robust and rules out anticipation factors. The touch-pressure detection threshold was calculated by averaging all turnaround points obtained (excluding responses obtained at the beginning of the experiment) after conversion of filaments numbering to pressure values. Turnaround points designate the points where the direction of intensity changes is reversed.

\subsection{Laser-evoked potentials (LEPS)}

Laser-evoked potentials were recorded with $\mathrm{CO}_{2}$ laser stimuli of $50 \mathrm{~ms}$ duration and a surface area of $80 \mathrm{~mm}^{2}$. The intensity was adjusted according to the threshold for "pricking pain" $(8.5-11 \mathrm{~mJ} /$ $\mathrm{mm}^{2}$ ) and short reaction times (300-600 ms), indicative for activation of Adelta $(A \delta)$ fibers. Whereas C-fiber stimulation of the hand area at detection threshold levels results in a sensation of nonpainful heat with a latency of around $800-1000 \mathrm{~ms}$, stimulation at A delta levels leads to a sensation of pricking pain with a faster onset (Opsommer and Plaghki, 2001). Each examination consisted of two sessions of about $30 \mathrm{~min}$, one exploring the symptomatic area with sensory disturbances and or neuropathic pain, and the other the contralateral body region mirroring the painful area. The order of testing in the two areas was randomized across subjects. A minimum of 30 and a maximum of 50 laser stimuli were delivered during each session, depending on the quality of the recordings (e.g. signal-to-noise ratio, ocular artifacts). Interstimulus interval was between 5 and $10 \mathrm{~s}$. To avoid habituation and or sensitization of the skin, the stimulus was slightly moved after each stimulus within the area of interest. For a detailed description of the experimental protocol we refer to Opsommer and Plaghki (2001). The procedure started with the assessment of the absolute detection thresholds for C-fiber and A $\delta$-fiber activation by the methods of limits. Ten to 15 stimuli were used for each threshold determination. Subjects were instructed to push a handhold button as quickly as possible at the first sensation felt.

The LEP recording session started with familiarization of the patient with the recording equipment and the stimuli that were going to be used. Following the measurement of the baseline skin temperature and the assessment of the thresholds for C-fiber and A $\delta$-fiber stimulation, LEP recordings started. In order to avoid fatigue and lack of concentration, each area was only tested once (the total duration of the QST and LEP procedures was close to $3 \mathrm{~h}$ ). Supraliminal (threshold $+25 \%$ ) stimuli were used for activating A $\delta$-nociceptors. In order to keep attention optimal and to limit as much as possible habituation to stimulation, laser stimuli were presented in maximum five blocks of 10 supraliminal stimuli. A 30-s time interval separated two successive blocks. LEPs were measured with a 21 channel EEG system (PL-EEG, Walter Graphtek, Germany), band pass $0.01-70 \mathrm{~Hz}$, sampling rate $167 \mathrm{cps}$. LEP's were processed off-line with Brain Vision Analyzer (Brain Products
GmbH, Germany) and EEGLAB (Delorme and Makeig, 2004). The signal was segmented in $3 \mathrm{~s}$ epochs $(-500$ to $+2500 \mathrm{~ms})$. A digital bandpass filter of $0.5-20 \mathrm{~Hz}$ was used. The low pass filter was set to $20 \mathrm{~Hz}$ because the acquisition rate was $167 \mathrm{cps}$ and the greatest part of signal power was in the delta-theta band, i.e. below $8 \mathrm{~Hz}$. Sweeps showing artifacts produced by ocular movements were rejected. Baseline correction (reference interval -0.5 to $0 \mathrm{~s}$ ), averaging and topographic mapping were carried out on the acquired traces. Three distinct components (N1, N2, P2) were characterized for each subject and within each LEP waveform recorded at the vertex lead, corresponding to $\mathrm{Cz}$ (Hatem et al., 2007). The N1 component is maximal at the contralateral temporal leads and should be referenced to the frontal leads. This was not done since we did not measure this tiny component. Latencies (in ms) were measured from stimulus onset to peak. LEP amplitude was measured from the N2 peak to the P2 peak.

\subsection{Positron Emission Tomography (PET) imaging}

An ECAT Exact HR + positron emission tomograph scanner (Siemens/CTI 961, Knoxville, Tn, USA) was used to measure changes in regional cerebral blood flow (rCBF). Prior to the emission tomograms, a ${ }^{68} \mathrm{Ga} /{ }^{68} \mathrm{Ge}$ transmission scan was acquired in 2D mode for attenuation correction. Emission tomograms were obtained in 3D mode after bolus injection of $300 \mathrm{MBq}(5 \mathrm{ml}) \mathrm{H}_{2}{ }^{15} \mathrm{O}$. The $60 \mathrm{~s}$ data acquisition frame started as soon as the intracranial radioactivity count rate rose sharply, i.e. usually $40-60 \mathrm{~s}$ after injection. PET images were reconstructed using filtered back-projection and Hann. filtering (cut-off frequency $=0.15$ cycles $/$ pixel, pixel size $=3.4 \mathrm{~mm}$ ).

Subjects were scanned twice, once following a period of nonusage of PNS, when the original pain and allodynic responses had reappeared (PNS-OFF), and once following a period of normal usage of PNS (PNS-ON). Depending upon the duration of the post-stimulation analgesic effect (data from the intake assessment and the home-evaluation), patients were asked to switch off their stimulator at a predetermined time before the start of the scanning session. The two sessions were randomized across patients. During each session, patients were scanned in the following three conditions, each repeated three times in a semi-randomized order:

i. Following a period of non-usage of PNS.

1. Baseline (pain): patients were scanned at rest and no stimulation was applied. Since patients had their stimulators switched off for an extended period, this condition was associated with spontaneous pain.

2. Allodynic stimulation: patients were scanned during allodynic thermal stimulation of the affected side. Periods of $5 \mathrm{~s}$ of thermal stimulation were alternated with $2 \mathrm{~s}$ periods of rest. The parameters for allodynic stimulation were based upon the results of a QST assessment preceding the PET examination.

3. PNS stimulation: patients were scanned after switching on their stimulator. The PNS stimulator was switched on $10 \mathrm{~s}$ before the start of the scan and continued throughout the 60-s scanning window. The patient's habitual stimulation parameters were used. In order to prevent an analgesic (after-)effect, the stimulator was switched off immediately when the scan was finished.

ii. Following a period of normal usage of PNS.

1. Baseline (pain-free): patients were scanned at rest and no stimulation was applied. Since patients had been using their stimulator normally in the days preceding the scanning session, this condition was associated with reduced or no spontaneous pain at rest. 
2. Non-painful thermal stimulation: patients were scanned during non-painful thermal stimulation of the affected side. The same stimulus intensity was used as in the allodynic stimulation condition. Since patients had been using their stimulator in normal therapeutic mode, the evoked sensation was non-painful in nature.

3. Painful stimulation: patients were scanned during noxious thermal stimulation of the affected limb. Like in the other sensory stimulation conditions, periods of $5 \mathrm{~s}$ of stimulation were alternated with $2 \mathrm{~s}$ periods of rest.

After each scan, patients had to rate pain intensity and pain unpleasantness on a 10 -point rating scale with 0 indicating no pain/not unpleasant and 10 the most intense pain/unpleasant sensation imaginable. Table S1 gives an overview of the different scanning conditions.

Since patients were implanted with MRI-incompatible implanted pulse generators, no MRI scans were acquired out of safety reasons for the patient and to avoid damage to the implanted material. For warping of the PET data into MNI space we therefore used the PET template provided in SPM5. Voxel counts were normalized by proportional scaling based on the global counts to correct for differences in the injected dose. Images were smoothed with a 3D-Gaussian kernel with a FWHM of $12 \mathrm{~mm}$ and the voxel size in MNI space was $2.0 \times 2.0 \times 2.0 \mathrm{~mm}$. Statistical analysis of the PET data was done by SPM5 (Wellcome Department of Imaging Neuroscience, UCL, London, UK). Data from patients with rightsided pain were left-right flipped and analyzed together with data from patients with left-sided pain. Main effects were calculated for the contrasts "PNS stimulation vs. rest", "allodynic stimulation vs. sensory stimulation" and "baseline (PNS-OFF) vs. baseline (PNSON)". Statistical maps were thresholded at $P<0.05$, false-discovery-rate (FDR)-corrected.

\section{Results}

\subsection{Description of the clinical cases}

The average age of the patients was $60.8 \pm 4.5$ years. The average duration of pain complaints at the time of the initiation of PNS therapy was $15.2 \pm 15$ years (range: $2-33$ years). All patients were treated for pain complaints in the upper limbs. Three patients suffered pain in the left hand (two cubital, one radial distribution), two from pain in the right hand (one radial and one cubital distribution). Average number of years of PNS implant at the time of the examination was $23.2 \pm 5.0$ years (range: $18-29$ years). Two of the patients used the stimulator daily, one weekly, one monthly and another one only "occasionally". A detailed description of the five cases is provided in Online supplementary data.

\subsection{Results home evaluation pain and quality-of-life}

The results of the effect of PNS on measures of clinical pain and daily functioning have been described before in detail (Van Calenbergh et al., 2009) and will here only be briefly summarized. Average pain intensity and pain unpleasantness ratings in the home environment were significantly lower during the three consecutive days with the stimulator on. The pain ratings dropped from $3.5 \pm 0.4$ and $3.8 \pm 0.4$ (respectively intensity and unpleasantness) during days without stimulation to $1.8 \pm 0.4$ and $1.8 \pm 0.4$ during days of normal usage of the stimulator $(P<0.05)$. Patients also rated the degree to which pain interfered with five variables - such as physical activities, mood, relations with others and enjoyment of life - on a VAS scale from 0 (not at all) to 10 (to an extreme degree). Average scores were significantly lower during the PNS-ON $(2.6 \pm 1.2)$ compared to the PNS-OFF period $(8.3 \pm 2.4)$.

\subsection{QST results}

The QST results are shown in Tables 2 and 3. Because of the small number of subjects and the large intersubject variability, individual responses are shown. All data are transformed in $z$-scores (Rolke et al., 2006b), based upon the results obtained in a sex- and agematched control population of 21 healthy subjects (mean age: $47 \pm 15.6$; range $20-71$ ). A negative $z$-score of -1 or -2 implies that the patient's score is respectively 1 or 2 standard deviations below the average score of the control population, whereas a positive $z$-score of 1 or 2 implies that his score is respectively 1 or 2 standard deviations above that of the control population. The QST data confirmed that patients had abnormally low CDTs and high WDTs, not only in the painful area but also in the mirror area (Table 2). Two patients had abnormally high and another two had abnormally low CPTs. Three patients had an abnormally high and one an abnormally low HPT in the painful area. PNS had no overall effect on CPTs or HPTs, either in the painful or in the mirror area. For HPT, three patients did not show a change in the painful area with PNS-ON and OFF, one patient showed a lower HPT during the on period and one patient (VBM) showed an increase in HPT. This patient also showed a lower CPT during stimulation. This confirms earlier findings (Kupers and Gybels, 1998) obtained in the same patient when

Table 2

Thermal detection thresholds ( $z$-scores).

\begin{tabular}{|c|c|c|c|c|c|c|c|c|}
\hline \multirow[t]{3}{*}{ Patient } & \multicolumn{2}{|c|}{ Painful area } & \multicolumn{2}{|c|}{ Mirror area } & \multicolumn{2}{|c|}{ Painful area } & \multicolumn{2}{|c|}{ Mirror area } \\
\hline & Off & On & Off & On & Off & On & Off & On \\
\hline & \multicolumn{4}{|c|}{ Cold detection threshold controls: $30.9 \pm 0.45{ }^{\circ} \mathrm{C}$} & \multicolumn{4}{|c|}{ Warmth detection threshold controls: $32.4 \pm 0.6^{\circ} \mathrm{C}$} \\
\hline VBM & -6.78 & -5.44 & -4.56 & -3.00 & 6.00 & 5.17 & 3.17 & 3.67 \\
\hline DRR & -8.56 & -0.22 & 0.11 & -0.11 & 3.08 & 9.58 & 3.50 & 2.00 \\
\hline DBH & -1.89 & -2.11 & -1.44 & -3.67 & 6.67 & 5.08 & 10.33 & 7.17 \\
\hline HUV & -3.11 & -4.00 & -3.33 & -1.89 & 2.00 & 2.42 & 3.83 & 3.17 \\
\hline VDB & 0.89 & 0.00 & -0.67 & -0.89 & 0.92 & 1.08 & 1.17 & 2.50 \\
\hline \multirow[t]{2}{*}{ Median } & -3.11 & -2.11 & -1.44 & -1.89 & 3.08 & 5.08 & 3.50 & 3.17 \\
\hline & \multicolumn{4}{|c|}{ Cold pain threshold controls: $12.0 \pm 4.85^{\circ} \mathrm{C}$} & \multicolumn{4}{|c|}{ Heat pain threshold controls: $43.2 \pm 2.60{ }^{\circ} \mathrm{C}$} \\
\hline VBM & 1.89 & 0.44 & -2.46 & -1.93 & -1.00 & 0.85 & 0.73 & 1.38 \\
\hline DRR & 2.13 & 2.51 & 2.44 & 2.13 & 2.19 & 2.27 & -0.04 & 0.62 \\
\hline DBH & -2.46 & -2.46 & -2.03 & -1.87 & 2.38 & 2.04 & 1.77 & 2.08 \\
\hline HUV & -2.46 & 2.42 & 2.38 & 2.15 & 2.04 & 0.62 & 0.12 & -1.73 \\
\hline VDB & 2.07 & 3.47 & -0.30 & 2.15 & -2.73 & -2.54 & 0.27 & 0.27 \\
\hline Median & 1.89 & 2.42 & -0.30 & 2.13 & 2.04 & 0.85 & 0.27 & 0.62 \\
\hline
\end{tabular}


Table 3

Touch sensitivity thresholds ( $z$-scores).

\begin{tabular}{|c|c|c|c|c|}
\hline \multicolumn{5}{|c|}{ Threshold healthy control population: $6.71 \pm 3.1 \mathrm{~g} / \mathrm{mm}^{2}$} \\
\hline \multirow[t]{2}{*}{ Patient } & \multicolumn{2}{|c|}{ Painful area } & \multicolumn{2}{|c|}{ Mirror area } \\
\hline & Off & On & Off & On \\
\hline VBM & -0.65 & -0.18 & -0.52 & -0.11 \\
\hline DRR & -0.63 & -0.53 & -0.68 & -0.65 \\
\hline $\mathrm{DBH}$ & 0.24 & 1.00 & -0.68 & 0.48 \\
\hline HUV & -0.63 & -0.63 & -0.65 & -0.66 \\
\hline VDB & -0.40 & -0.47 & -0.42 & -0.19 \\
\hline Median & -0.63 & -0.47 & -0.65 & -0.19 \\
\hline
\end{tabular}

tested more than 10 years ago (Fig. S1, see the online version at 10.1016/j.ejpain.2010.06.015).

The results of the von Frey testing is shown in Table 3. Touch detection thresholds were in general slightly lower than in a control population, both in the painful zone and in the pain mirror area. In two subjects, there was a clear tendency for an increase in touch thresholds during the PNS-ON period, both in the painful area and in the mirror area.

\subsection{LEP results}

The detection rate of supraliminal stimuli for A $\delta$-nociceptors was on average $97 \%$, exception made for patient DBH who presented an average detection rate of $43 \%$. Reaction times were around $500-600 \mathrm{~ms}$, which is in the range for $A \delta$-fiber responses. Although reaction times seemed a bit shorter for the painful compared to the mirror control area, this did not reach statistical significance. PNS had no effect on reaction times.

In all patients, the N2-P2 complexes of LEP recordings were of rather low amplitudes (Table 4). The latencies of the N2 and P2 components varied much between subjects but there were no systematic deviations from the control group neither between painful and mirror sites nor between PNS-OFF and ON conditions in both sites (data not shown). However, when comparing the N2-P2 amplitudes in the two conditions, there was a small but similarly significant increase in amplitude in the PNS-ON condition for both sites (Wilcoxon matched pairs test, $z=-2.023, P=0.043$ ).

\subsection{PET results}

The results of the PET study are shown in Figs. 1-3 and in Tables S2-S4. Average pain ratings in the allodynic and non-painful heat condition were respectively $5.4 \pm 3.2$ and 0 . Spontaneous pain ratings in the PNS-OFF and ON period were respectively $3.0 \pm 1.7$ and $0.4 \pm 1.1$. Fig. 1 and Table S2 show the results of the contrast "allodynic stimulation versus non-painful heat stimulation". It is important to recall that the physical stimulus used in the two conditions is identical. During PNS-ON, thermal stimulation evoked signifi-

Table 4

LEP (N2-P2) amplitudes ( $z$-scores).

\begin{tabular}{llllll}
\hline \multicolumn{3}{l}{ N2-P2 amplitude healthy control population: $30.1 \pm 16.2 \mu \mathrm{V}$} \\
\cline { 1 - 3 } Patient & \multicolumn{3}{l}{ Painful area } & & Mirror area \\
\cline { 2 - 3 } \cline { 5 - 6 } & Off & On & & Off & On \\
\hline VBM & -1.23 & -0.98 & & -0.78 & -0.50 \\
DRR & -1.44 & -0.36 & & -0.80 & -0.55 \\
DBH & -1.30 & -1.07 & & -1.30 & -0.86 \\
HUV & -1.06 & -1.03 & & -1.10 & -1.05 \\
VDB & -1.00 & 0.01 & & -0.67 & -0.37 \\
Median & -1.23 & -0.98 & & -0.80 & -0.55 \\
\hline
\end{tabular}

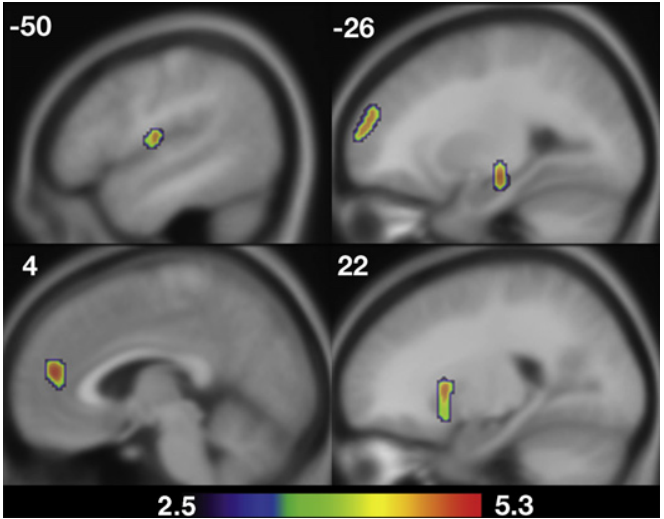

Fig. 1. PET data showing areas where rCBF was significantly higher during allodynic as compared to non-painful heat stimulation. Data are projected on sagittal slices. Colored voxels represent clusters of significant rCBF signal increases ( $T$-test, $P<0.05$, FDR-corrected; the color scale denotes the cluster $T$-values) during the PNS-OFF period. The numbers refer to the lateral-medial orientation ( $x$-coordinate, negative numbers indicate the left side of the brain) of the slices in Montreal Neurological Institute (MNI) space. The same thermal stimulus was applied in the two conditions, resulting in the sensation of allodynia in the PNS-OFF period and a non-painful sensation in the PNS-ON period. PNS stimulation reduced rCBF responses in the ACC, insula, prefrontal cortex, caudate nucleus and hippocampus.

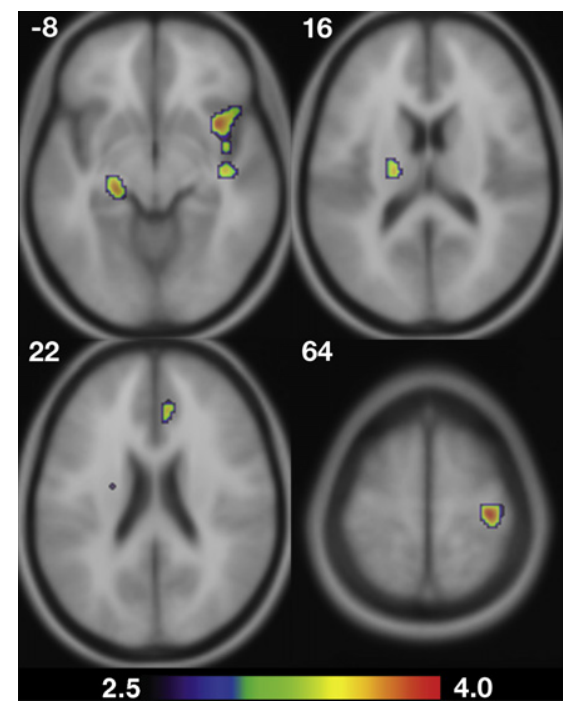

Fig. 2. Increases in rCBF produced by PNS (contrast "PNS versus baseline-OFF". Data are shown on axial slices. Colored voxels represent clusters of significant rCBF signal increases ( $T$-test, $P<0.05$, FDR-corrected; the color scale denotes the cluster $T$ values). The numbers refer to the dorso-ventral orientation ( $z$-coordinate) of the slices in MNI space. PNS induced rCBF increases in SI, ACC, thalamus and rostral inferior insula. The left part of the brain is shown to the left of the image.

cantly smaller rCBF responses in several parts of the "pain matrix", more specifically in the rostral anterior cingulate cortex, dorsolateral prefrontal cortex, posterior insula and lenticular nucleus. No significant $\mathrm{rCBF}$ changes were observed in the thalamus, SI and SII. However, when using a more lenient statistical threshold, rCBF changes were also detected in posterior thalamus and SII. Relatively higher $\mathrm{rCBF}$ was measured in the insula, subgenual cingulate, posterior thalamus and nucleus accumbens when allodynic stimulation was compared with painful stimulation (data not shown). Fig. 2 and Table S3 show the changes in rCBF produced by PNS. A strong $\mathrm{rCBF}$ increase occurred in the contralateral primary somatosensory cortex (SI), corresponding with the hand area. Additional rCBF increases were observed in the antero-ventral insula, the thalamus and the rostral anterior cingulate cortex. When lowering the 


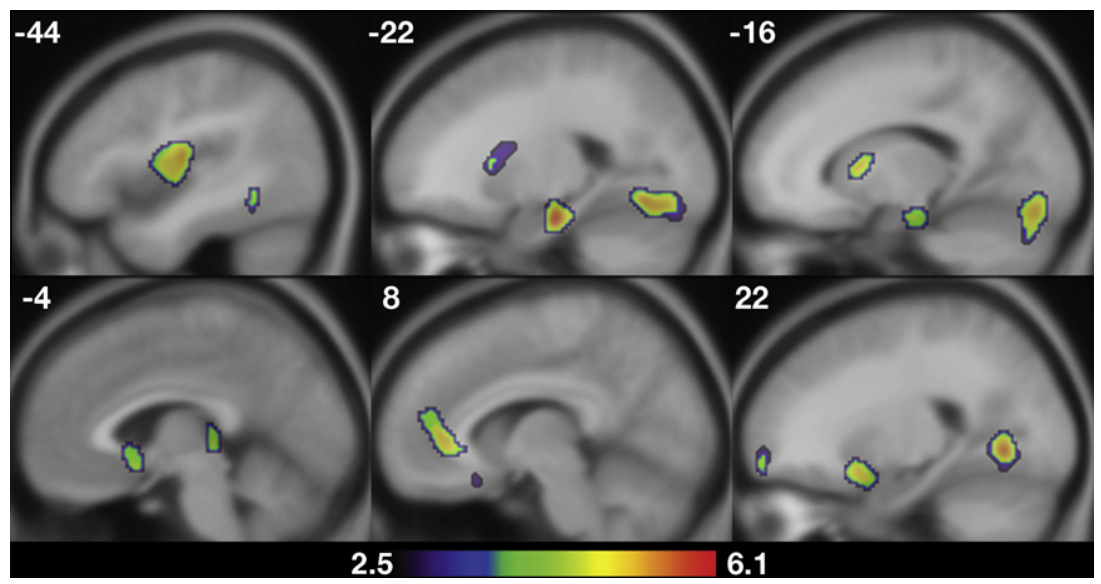

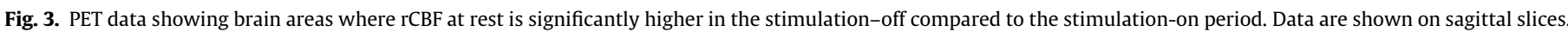

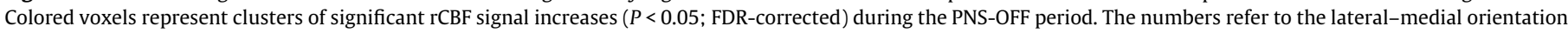

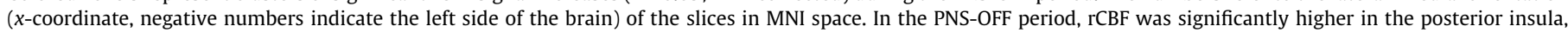
perigeniculate and subgenual cingulate cortex, posterior thalamus, caudate nucleus and prefrontal cortex.

statistical threshold to $P<0.001$, uncorrected, an rCBF increase was also observed in the right inferior parietal lobule (Brodmann Area (BA) $40 ; x=32, y=-40, z=44$ ) and the orbital gyrus (BA 11; $x=18, y=32, z=-26)$. Fig. 3 and Table S4 show the rCBF changes when comparing the rest conditions in the pain-reduced (PNS-ON) and pain (PNS-OFF) state. During the pain-reduced state, resting state $\mathrm{rCBF}$ was significantly lower in the rostral anterior and subgenual cingulate (adjoining the nucleus accumbens) cortices, posterior thalamus (pulvinar), posterior insula, caudate nucleus, and the right inferior (BA 47) and superior (BA 10) frontal gyri. No changes in $\mathrm{rCBF}$ across the two conditions were observed in the lateral pain system. However, when using non-corrected $P$-values, rCBF changes were also detected in SI and SII.

\section{Discussion}

We present here five case reports of patients with mononeuropathic pain who were treated for more than 20 years with direct electrical peripheral nerve stimulation. This is the first comprehensive study to measure in an objective and multimodal manner the therapeutic efficacy of PNS, its effect on somatosensory processing and its underlying neurophysiological mechanisms. We believe the data presented in this paper are novel and encouraging but at the same time we are also fully aware of the limitations of our data. First, this is an open study without a placebo control condition, which means that it is impossible to rule out with certainty that some of the reported therapeutic effects are not due to placebo effects or experimenter-demand effects. The second limitation concerns the small number of subjects which reduces the statistical power of the group data. We were only able to include five patients out of the ten we initially contacted. Five out of the original 11 patients no longer used PNS. It could therefore be argued that our study is biased by the selection of patients still using the stimulator. However, four of the five explants were for technical reasons or hardware infections and not because of lack of analgesic effect. These patients were successfully treated with SCS after removal of the PNS device. Finally, an important limitation is the variability in the amount of time the patients actively used PNS, some on a daily basis and others at weekly intervals. This variability has been also reported for other neurostimulation techniques such as SCS and DBS (Gybels et al., 1990). Despite these limitations, there are a number of objective findings which warrant further prospective studies such as the convergence of the clinical reports of analgesic efficacy and the PET findings of reduced activation within the pain matrix, and the convergence of some of the PET and LEP data.

\subsection{Effects of PNS on clinical pain}

Patients were asked to evaluate their spontaneous pain and measures of quality-of-life in their home environment during 3 days of normal usage of the stimulator and during 3 days of refraining from stimulation. The clinical data have been described in detail elsewhere (Van Calenbergh et al., 2009) and will therefore be described only briefly. Patients reported lower pain ratings and improved quality-of-life measures during the days with PNS. This is in line with their verbal reports of satisfaction with PNS expressed prior to the onset of the study. As mentioned above, placebo effects or experimenter-demand effects cannot be ruled out.

\subsection{Thermal and tactile perception}

Compared to an age- and sex-matched control group, our patients had abnormal cool and warmth thresholds, not only in the painful area but also in the mirror area. In addition, all patients had abnormally low or high heat and cold pain thresholds. Together, this confirms the neuropathic character of the pain complaints (Rolke et al., 2006a). Touch detection thresholds in the patient were not significantly different from those of the control group.

At the group level, PNS had no significant effect on thermal perception. Both the thresholds for cool and warmth and cold pain and heat pain were not significantly altered by PNS. The lack of effect on cool and warmth detection may not come as a surprise as PNS exerts primarily an effect on nociceptive processing. However, the lack of a significant effect on heat and cold pain thresholds was unexpected. Several explanations may be evoked for these negative findings. First, the patient sample size is small, making it hard to show any significant effect. Next, looking at the individual data, we notice a large intersubject variability. As the pain thresholds were determined at the end of a LEP and QST session lasting about $3 \mathrm{~h}$, fatigue or concentration difficulties may have lead to inconsistent responses in some patients. Finally, the method of assessing pain responses during the QST sessions may also have lead to the negative results. We used a simple methods of limits to measure pain threshold and did not test for responses to suprathreshold stimuli. During the extra QST session preceding the PET investigation, we used the method of constant stimuli instead to assess pain 
responsiveness. Using an ascending-descending staircase method, participant's responses were tested to $5 \mathrm{~s}$ lasting thermal stimuli. The reason for doing so was to assure that participants would tolerate the pain stimuli in the ensuing PET examination in which we also applied $5 \mathrm{~s}$ lasting thermal stimuli. The temperatures which were consequently used during the PET examination were $1{ }^{\circ} \mathrm{C}$ below the pain threshold as assessed by the methods of limits. Nonetheless, they were described as painful by the patients in the PNS-OFF condition, probably due to temporal summation.

\subsection{LEPS data}

It is well recognized that LEPs provide a sensitive and reliable tool for exploring the functional status of the thermo-nociceptive pathways within the somatosensory system (Treede et al., 2003; Truini et al., 2004). LEP abnormalities consist of complete absence, amplitude reductions and/or latency increases. The LEP data are complementary to our QST data as the former reflect mainly responses to A $\delta$ nociceptor activation whereas the latter reflect responses to C-nociceptor activation by slow ramped thermal stimuli. The main findings are the low amplitudes of LEPs and the increase of LEP amplitude in the PNS-ON condition. Indeed, all patients presented rather low amplitudes of the N2-P2 complex and this was true for the painful and mirror side. At first sight, this may result from the higher age of the patients since LEP amplitude declines significantly with age (Gibson et al., 1991; Truini et al., 2004). However, the patients' N2-P2 amplitudes were compared to those of an age-matched control group. It is therefore tempting to explain the reduced LEP amplitude as resulting from a chronic neuropathic pain condition. As stated by Garcia-Larrea and co-workers (2002), chronic pain coupled with reduced LEPs substantiates the diagnosis of neuropathic pain, whereas the finding of normal or enhanced LEPs to stimulation of a painful territory suggests the integrity of pain pathways, and does not support a neuropathic pathophysiology. This is further evidenced by the association of the pain relieving effect of PNS and a small but significant increase of LEP amplitude in the PNS-ON condition. Indeed, a similar effect was reported by Sestito and colleagues with SCS in patients with cardiac syndrome X (Sestito et al., 2008). These authors hypothesized that SCS removes the inhibitory influence that the chronic cardiac pain exerts on the nociceptive system of these patients through a counter-irritation mechanism. Similarly, in the present group of patients, the pain relieving effect of PNS may have reduced the counter-irritation mechanism and the consequent nociceptive inhibition, leading to an increased LEP amplitude.

\subsection{PET data}

According to the original theory by Melzack and Wall (1965), the therapeutic principle of PNS stimulation is based upon the activation of $A$ beta $(A \beta)$ fibers. This is confirmed by the present findings as PNS lead to a significant rCBF increase in the contralateral primary somatosensory cortex. The increased $\mathrm{rCBF}$ response in SI is also in line with our finding of larger LEP amplitudes during the PNS-ON condition. Interestingly, previous studies on the analgesic effects of SCS and DBS failed to show increased activation in SI (Davis et al., 2000; Duncan et al., 1998; Hautvast et al., 1997; Kupers et al., 2000), suggesting that the analgesic mechanisms of PNS are at least partly different from those of SCS or DBS. Alternatively, the stimulation-induced $\mathrm{rCBF}$ increase in SI may simply represent an epiphenomenon of large fiber stimulation, unrelated to the analgesic effect. In addition to activation of SI, PNS stimulation also lead to an increase in rCBF in the antero-ventral insula, thalamus and ACC, suggesting that besides activation of the dorsal column medial lemniscal system (Nathan and Wall, 1974), other mechanisms may play a role in the analgesic effects of PNS. Several of these areas have been shown to be involved in the modulation of pain (Davis et al., 2000; Duncan et al., 1998; Peyron et al., 2007; Starr et al., 2009). For instance, the ACC is activated during DBS (Davis et al., 2000; Duncan et al., 1998), motor cortex stimulation (Peyron et al., 2007) and other analgesic procedures (Peyron et al., 2000). Comparing the baseline scans during the PNS-ON and -OFF period revealed that during the period of active stimulation, resting state rCBF was significantly smaller in several parts of the "pain matrix", including the posterior thalamus and insula, perigeniculate and subgenual cingulum, caudate nucleus and orbitofrontal cortex. This was associated with lower spontaneous pain ratings during the PNS-ON period. The comparison of the response to innocuous thermal stimulation during the active and non-active stimulation period reveals that cerebral blood flow responses were smaller in the ACC, posterior insula, caudate nucleus and prefrontal cortex. Together, these data show that PNS reduces spontaneous and heat-induced activity within the "pain matrix" and increases activation in areas involved in pain modulation. We suggest that these local haemodynamic changes reflect the areas mediating the analgesic effects of PNS.

In conclusion, the present unique series of case reports with more than 20 years of follow-up suggest that PNS can provide long-term relief in patients with peripheral neuropathic pain following peripheral nerve lesions, with few side-effects or complications. The current data certainly do not form the final proof of efficacy of PNS. However, they incite in envisioning prospective randomized case-control trials in order to show the therapeutic efficacy of PNS in a more unequivocal manner.

\section{Disclosures}

This study was partly sponsored by a grant from Medtronic Europe. None of the authors has any shares or financial interest with Medtronic. This manuscript has been written completely independently and has not been sent out or discussed with any Medtronic representative or employee for approval before submission.

\section{Acknowledgments}

The authors wish to express their thanks to Stijn Dirix (UZ Gasthuisberg) for expert technical and practical help during the planning of the PET studies and to members of the Leuven PET radiochemistry team for tracer preparations. KVL is senior clinical investigator for the Fund for Scientific Research Flanders (FWO).

\section{Appendix A. Supplementary material}

Supplementary data associated with this article can be found, in the online version, at doi:10.1016/j.ejpain.2010.06.015.

\section{References}

Burns B, Watkins L, Goadsby PJ. Treatment of intractable chronic cluster headache by occipital nerve stimulation in 14 patients. Neurology 2009;72:341-5.

Davis KD, Taub E Duffner F, Lozano AM, Tasker RR, Houle S, et al. Activation of the anterior cingulate cortex by thalamic stimulation in patients with chronic pain: a positron emission tomography study. J Neurosurg 2000;92:64-9.

Delorme A, Makeig S. EEGLAB: an open source toolbox for analysis of single-trial EEG dynamics. J Neurosci Methods 2004;134:9-21.

Duncan GH, Kupers RC, Marchand S, Villemure JG, Gybels JM, Bushnell MC. Stimulation of human thalamus for pain relief: possible modulatory circuits revealed by positron emission tomography. J Neurophysiol 1998;80:3326-30.

Dyck PJ, O’Brien PC, Kosanke JL, Gillen DA, Karnes JL. A 4, 2 and 1 stepping algorithm for quick and accurate estimation of cutaneous sensation threshold. Neurology 1993;43:1508-12

Garcia-Larrea L, Convers P, Magnin M, André-Obadia N, Peyron R, Laurent B, et al. Laser-evoked potential abnormalities in central pain patients: the influence of spontaneous and provoked pain. Brain 2002;125:2766-81. 
ARTICLE IN PRESS

8

R. Kupers et al./European Journal of Pain $x x x$ (2010) $x x x-x x x$

Gibson SJ, Gorman MM, Hemle RD. Assessment of pain in the elderly using eventrelated cerebral potentials. In: Bond $\mathrm{MR}$, Charlton JE, Woolf CJ, editors. Proceedings of WIth world congress on pain. Elsevier Science; 1991. p. 527-33.

Gybes J, Kupers R, Van Calenbergh F. Physiological approaches of pain. In: Dimitrijevic Wall, Lindblom U, editors. Recent achievements in restorative neurology 3: altered sensation and pain. Basel: Karger; 1990. p. 79-86.

Gybes JM, Sweet WH. Neurosurgical treatment of persistent pain. Pain and headache, vol. 11. Basel: Karger; 1989.

Gybels J, Van Calenbergh F. The treatment of pain due to peripheral nerve injury by electrical stimulation of the injured nerve. Adv Pain Res Then 1990;13:217-22.

Hater SM, Plaghki L, Mouraux A. How response inhibition modulates nociceptive and non-nociceptive somatosensory brain-evoked potentials. Chin Neurophysiol 2007; $118: 1503-16$

Hautvast RW, Ter Horst GJ, DeJong BM, DeJongste MJ, Blanksma PK, Pans AM, et al. Relative changes in regional cerebral blood flow during spinal cord stimulation in patients with refractory angina pectoris. Eur J Neurosci 1997;9:1178-83.

Kupers R, Gybels J. Evaluation of pain surgery. In: Gildenberg P, Taker R, editors. Textbook of stereotactic and functional neurosurgery. Mc Grow Hill; 1998. p. 1311-9.

Kupers RC, Gybels JM, Gjedde A. Positron emission tomography study of a chronic pain patient successfully treated with somatosensory thalamic stimulation. Pain 2000;87:295-302.

Kupers RC, Van den Over R, Van Houdenhove B, Vanmechelen W, Hep B, Nuttin B, et al. Spinal cord stimulation in Belgium: a nation-wide survey on the incidence, indications and therapeutic efficacy by the health insurer. Pain 1994;56:211-6. Melzack R, Wall PD. Pain mechanisms: a new theory. Science 1965;150:971-9.

Mouraux A, Iannetti GD. Nociceptive laser-evoked brain potentials do not reflect nociceptive-specific neural activity. J Neurophysiol 2009;101:3258-69.

Mobs RJ, Nair S, Blum P. Peripheral nerve stimulation for the treatment of chronic pain. J Chin Neurosci 2007;14:216-21.

Nathan PW, Wall PD. Treatment of post-herpetic neuralgia by prolonged electric stimulation. Br Med J 1974;3:645-7.

Opsommer E, Plaghki L. Maturational changes in the thermoalgesic system in humans from childhood to adulthood revealed by $\mathrm{CO}_{2}$ laser evoked brain potentials following cutaneous heat stimuli. Neurosci Lett 2001;316:137-40.
Peyron R, Faillenot I, Mertens P, Laurent B, Garcia-Larrea L. Motor cortex stimulation in neuropathic pain. Correlations between analgesic effect and hemodynamic changes in the brain. A PET study. Neurolmage 2007;34:310-21.

Peyron R, Laurent B, Garcia-Larrea L. Functional imaging of brain responses to pain: a review and meta-analysis. Neurophysiol Chin 2000;30:263-88.

Rolke R, Baron R, Maier C, Tölle TR, Treede RD, Beyer A, et al. Quantitative sensory testing in the German Research Network on Neuropathic Pain (DFNS): standardized protocol and reference values. Pain 2006a;123:231-43.

Rolke R, Magerl W, Campbell KA, Schalber C, Caspari S, Birklein F, et al. Quantitative sensory testing: a comprehensive protocol for clinical trials. Eur J Pain 2006b;10:77-88.

Sestito A, Lanza GA, Le Pera D, De Arras L, Sgueglia GA, Infusino F, et al. Spinal cord stimulation normalizes abnormal cortical pain processing in patients with cardiac syndrome X. Pain 2008;139:82-9.

Slavin KV. Peripheral nerve stimulation for neuropathic pain. Neurotherapeutics 2008;5:100-6.

Slavin KV, Burchiel KJ. Use of long-term nerve stimulation with implanted electrodes in the treatment of intractable craniofacial pain. J Neurosurg 2000;92:576.

Starr CJ, Sawaki L, Wittenberg GF, Burdette JH, Oshiro Y, Quevedo AS, et al. Roles of the insular cortex in the modulation of pain: insights from brain lesions. J Neurosci 2009;29:2684-94.

Sweet WH. Control of pain by direct electrical stimulation of peripheral nerves. Chin Neurosurg 1976;23:103-11.

Treed RD, Lorenz J, Baumgärtner U. Clinical usefulness of laser evoked potentials. Neurophysiol Chin 2003;33:303-14.

Truini A, Romaniello A, Galeotti F, Iannetti GD, Cruccu G. Laser evoked potentials for assessing sensory neuropathy in human patients. Neurosci Lett 2004;361: 25-8.

Van Calenbergh F, Gybels J, Van Laere K, Dupont P, Plaghki L, Depreitere B, et al. Long term clinical outcome of peripheral nerve stimulation in patients with chronic peripheral neuropathic pain. Surg Neurol 2009;72:330-5.

Wall PD, Sweet WH. Temporary abolition of pain in man. Science 1967;155:108-9.

Please cite this article in press as: Kupers $\mathrm{R}$ et al. Multimodal therapeutic assessment of peripheral nerve stimulation in neuropathic pain: Five case reports

with a 20-year follow-up. Eur J Pain (2010), doi:10.1016/j.ejpain.2010.06.015 
ARTICLE IN PRESS

R. Kupers et al./European Journal of Pain $x x x$ (2010) $x x x-x x x$

9

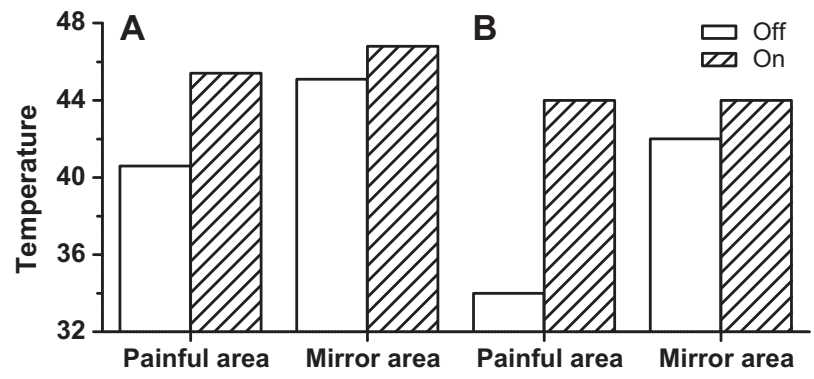

Fig. S1. QST data showing the effect of PNS on the pain threshold (median value of three assessments) for heat stimuli in the painful and contralateral hand in patient VBM tested on two different occasions. (A) Data from the current examination. The heat pain threshold in the painful area increased from $40.5^{\circ}$ (PNS-OFF) to $45.7^{\circ}$ (PNS-ON). There was a smaller increase in the contralateral hand. (B) Results from the same patient tested 10 years earlier. Results are very similar: PNS abolished the heat allodynic response in the painful area and only had a mild effect on the heat pain threshold at the contralateral side.

Please cite this article in press as: Kupers $\mathrm{R}$ et al. Multimodal therapeutic assessment of peripheral nerve stimulation in neuropathic pain: Five case reports with a 20-year follow-up. Eur J Pain (2010), doi:10.1016/j.ejpain.2010.06.015 